style attributes or a combination of both. Numerous studies in the disciplines of epidemiology, work physiology, psychology, and biochemistry have all pointed towards a beneficial effect of physical activity on health, but the optimal intensity, frequency, and duration of physical activity has yet to be established.

Although our study was observational, the men were randomly selected from a general population. The estimated effect of jogging did not depend on the inclusion of intermediate variables, supporting the association of jogging with lower mortality.

Whether light, moderate, or vigorous exercise should be recommended to the public has changed through the years. Although light exercise has some value, moderate and vigorous exercise is now considered more favourable for health. ${ }^{3}$ Our study supports this by showing that even a vigorous activity such as jogging is associated with a beneficial effect on mortality.

Contributors: PS initiated and conducted the study and contributed to the writing of the manuscript with PL. JP was responsible for the statistical analysis of the data and contributed to the writing. PS will act as guarantor for the paper.

Funding: Danish Heart Foundation.

Competing interests: None declared.

1 Appleyard M, Hansen AT, Schnohr P, Jensen G, Nyboe J. The Copenhagen City heart study. A book of tables with data from the first examination gen City heart study. A book of tables with data from the first examination (1976-78) and a five-ye
$1989 ; 170$ (suppl 41$): 1-160$.

2 Andersen PK, Borgan Oe, Gill RD, Keiding N. Statistical models based on counting processes. New York: Springer Verlag, 1993.

Fentem PH. Benefits of exercise in health and disease. BMJ 1994;308:1291-5.

4 Pate RR, Pratt M, Blair ST, Haskell WL, Macera CA, Bourchard C, et al. Physical activity and public health. JAMA 1995;273:402-7.

(Accepted 11 May 2000)

\title{
Is evidence based medicine neglected by royal college examinations? A descriptive study of their syllabuses
}

\author{
Wai-Ching Leung, Paula Whitty
}

Although the value of evidence based medicine has been debated, ${ }^{1}$ the benefits of teaching it to undergraduates $^{2}$ and postgraduates ${ }^{3}$ have been shown and have been acknowledged in the development of the new undergraduate medical curriculum. ${ }^{4}$ However, lack of adequate training is a major obstacle to postgraduates. $^{3}$

Evidence based medicine has four distinct steps ${ }^{5}$ : formulate clear clinical questions from a patient's problem; search the literature for relevant articles; evaluate the evidence for its validity and usefulness; apply useful findings in clinical practice.

This report examines the level of skills in evidence based medicine that are formally assessed by the royal colleges in the United Kingdom, through a review of the colleges' syllabuses for postgraduate examinations that are compulsory for specialist training.

\section{Methods and results}

We reviewed all syllabuses in effect on 20 October 1999 that were held by 16 faculties or royal colleges in the United Kingdom, representing 15 major specialties. Surgical examinations (held by three royal colleges) were reviewed separately. Subspecialties in surgery and pathology were grouped as a single specialty. Radiology and oncology, which are both examined by the Royal College of Radiologists, were analysed separately.

We reviewed each syllabus to determine whether the skills required for the four steps in evidence based medicine were assessed. The authors initially reviewed the syllabuses independently, and any disagreements (of which there were very few) were resolved by discussion. Where it was clear from the syllabus that a specific section of the examination is dedicated to examining skills in evidence based medicine, we obtained sample or past papers if available.

These skills were not substantially assessed in seven of a total of 17 syllabuses, for five out of 15 major specialties represented: general medicine, surgery, paediatrics, ophthalmology, and radiology. (We did not regard assessment of basic statistics alone as adequate for step three.) A dedicated section of the examination explicitly assesses these skills in five syllabuses (table). Most of these five syllabuses focus on the candidates' ability to evaluate the evidence for validity, but emphasis varies on the skills in evaluating the evidence for its usefulness, formulating a clear clinical question, searching for relevant literature, and implementing useful findings in clinical practice. In the remaining five syllabuses in which skills in evidence based medicine are mentioned (see tables on $B M J$ website), there are no dedicated procedures for examining these skills in anaesthetics, obstetrics and gynaecology, and oncology; in pathology and occupational health, candidates have to submit a dissertation with original research data.

\section{Comments}

One third of the specialties do not assess skills in evidence based medicine in their examination system. Examinations often exert a steering effect on the curriculum, and it would be difficult for future doctors to keep their professional knowledge and skills up to date unless these skills are learnt during training and are regularly applied in clinical practice.

Two thirds of the postgraduate examinations have no dedicated sections for the examination of skills in evidence based medicine. Even in syllabuses where these skills are examined, this is generally limited to skills in evaluating the evidence for validity. However,
Epidemiology and Public Health, Newcastle City Health Trust, Newcastle upon Tyne NE4 6BE Wai-Ching Leung senior registrar in public health medicine

Department of Epidemiology and Public Health, University of Newcastle upon Tyne, Newcastle upon Tyne NE2 $4 \mathrm{HH}$

Paula Whitty senior lecturer in medical care epidemiology Correspondence to: W-C Leung W.C.Leung@ncl. ac.uk

\section{BMJ 2000;321:603-4}

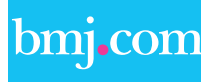

Tables showing examinations in which skills in evidence based medicine are and are not assessed are on the BMJ's website. 
Examinations with one or more dedicated sections for examining elements of skills in evidence based medicine explicitly

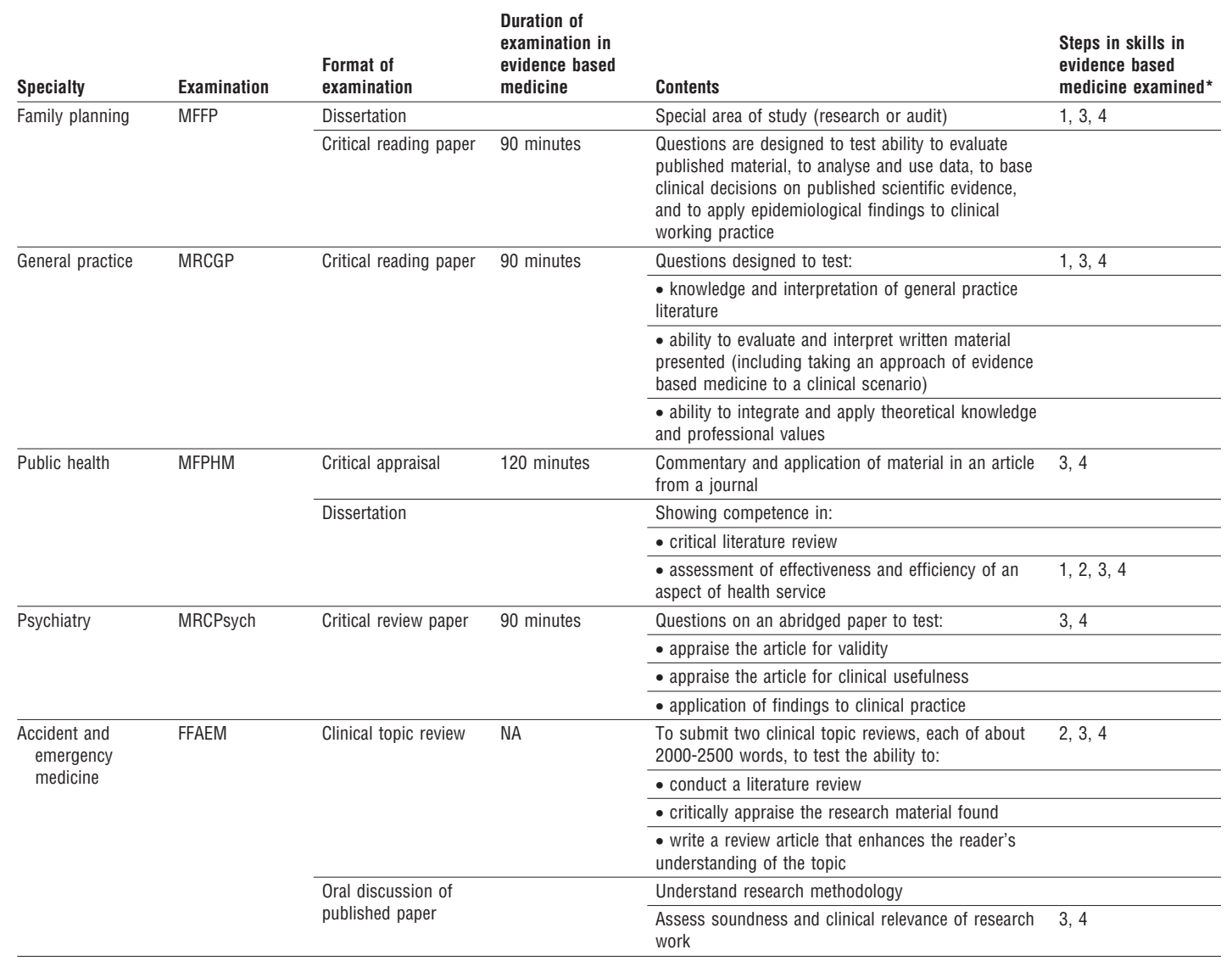

*The steps in evidence based medicine are: 1 Formulate clear clinical questions from a patient's problem. 2 Search the literature for relevant articles. 3 Evaluate the evidence for its validity and usefulness. 4 Apply useful findings in clinical practice.

to practise evidence based medicine effectively, skills in formulating a clear question, evaluating evidence for its usefulness, and applying findings to clinical practice are equally important. ${ }^{2}$

We acknowledge that royal college examinations are only one element of specialist training and that revision of some syllabuses may be under consideration. Nevertheless, the strategies of the royal colleges for assessing skills in evidence based medicine should be reviewed, possibly by following good examples of the few that assess these skills in some depth.

Contributors: PW initiated the study and contributed to the study design, analysis, and writing of the paper. WCL contributed to the study design, collation of the syllabuses, analysis, and drafting of the paper. WCL is the guarantor for the study.

Funding: None.

Competing interests: None declared.

1 Molesworth N. Sacred cows: to the abattoir! Down with EBM! BMJ 1998;317:1720-1.

2 Norman GR, Shannon SI. Effectiveness of instruction in critical appraisal (evidence-based medicine) skills: a critical appraisal. Can Med Assoc J 1998;158:177-81.

3 McAlister FA, Graham I, Karr GW, Laupacis A. Evidence-based medicine and the practising clinician.J Gen Intern Med 1999;14:236-42.

4 General Medical Council. Tomorrow's doctors: recommendations on undergraduate medical education. London: GMC, 1993.

5 Sackett DL, Richardson WS, Rosenberg WMC, Haynes RB. Evidence-based medicine. How to practise and teach EBM. Edinburgh: Churchill Livingstone, 2000.

(Accepted 4 May 2000)

\section{Email submissions from outside the United Kingdom}

We offer an email submission service for authors from outside the UK. The address is papers@bmj.com

Ideally our email server would link seamlessly with our manuscript tracking system, but for now it does not, which is why we are offering the service only to authors outside the UK. Most post in the UK arrives the next day, so UK authors have the least to gain in speed of delivery from email delivery. As soon as our systems improve we will invite email submissions from everyone.

If you choose to send your submission by email please would you send the text and any tables and figures as attached files, together with a covering letter giving all your contact details (postal address, phone, fax, and email address). We can read files created with most word processing, graphics, and spreadsheet programs.

When your submission is received in our email box you will receive an automatic acknowledgment to show that it has arrived. If the submission is incomplete we will contact you and ask you to resend the missing information.

Once the submission is complete we will register it on our manuscript tracking system and you will receive a standard acknowledgment in the post.

Letters to the editor should continue to be sent direct to bmj.com as rapid responsesor toletters@bmj.com 